
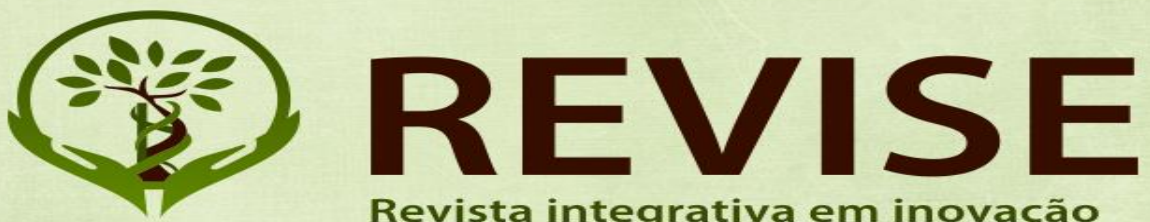

Revista integrativa em inovação tecnológica nas ciências da saúde

\title{
Artigo para discussão: \\ AVALIAÇÃO IN VITRO DO EXTRATO HIDROALCOÓLICO E AQUOSO DE GENGIBRE (Zingiber officinale [WILLD.] ROSCOE) SOBRE O FUNGO Lasiodiplodia theobromae
}

\author{
Lavínia dos Santos Mascarenhas \\ Monica Junqueira Machado \\ Noelma de Brito Miranda \\ Vânia de Jesus Santos Oliveira \\ Pedro Henrique Ribeiro Santana
}

FAMAM

\begin{abstract}
RESUMO
O objetivo é buscar métodos alternativos ao uso de defensivos agrícolas nas plantações agrícolas de grande escala. Assim, na tentativa de encontrar uma alternativa ao controle de doenças no campo, menos agressivo, surge métodos alternativos com extratos de plantas que apresentam propriedades antifúngicas. Dentre estas plantas, se encontra o gengibre, uma espécie de importância medicinal, aromática e condimentar que pertencente à família Zingiberaceae. Questiona-se se a aplicação dos extratos hidroalcoólico e aquoso de gengibre (Zingiberofficinale [WILLD.] Roscoe) podem interferir nas atividades fisiológicas do fungo Lasiodiplodia theobromae in vitro. A metodologia de pesquisa foi exploratória, descritiva e qualitativa/quantitativa. O trabalho foi realizado nos Laboratórios Múltiplo I e no de Ciências Biológicas da Faculdade Maria Milza (FAMAM), no campus de Governador Mangabeira. O isolado fúngico utilizado na pesquisa foi obtido a partir de lesões características da podridão da raiz da mandioca. $\mathrm{O}$ isolado foi conservado em placas de Petri contendo o meio BatataDextrose-Ágar (BDA) em geladeira. As análises realizadas nesta pesquisa mostraram que o extrato hidroalcoólico e aquoso de gengibre nas concentrações acima de $15 \%$ mostraram as maiores interferências no crescimento, na esporulação e germinação de conídios do fungo, impedindo o seu desenvolvimento in vitro. Em virtude destes resultados promissores, seria interessante a realização de outras análises levando em consideração este fator sistema (extrato de gengibre e o fungo L. theobromae) analisando outros parâmetros que possam servir de base confirmatória da ação fungitóxica do extrato de gengibre.

Palavras-Chaves: Agrotóxico. Intoxicações. Extratos de planta. Gengibre. Fitoterápico.
\end{abstract}



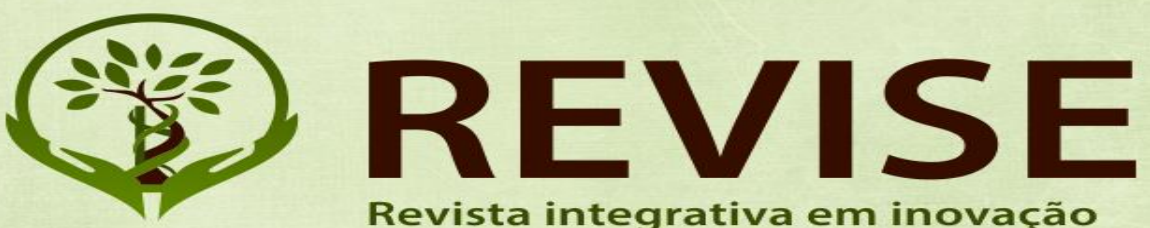

Revista integrativa em inovação tecnológica nas ciências da saúde

\title{
IN VITRO EVALUATION OF GINGER (Zingiber officinale [WILLD. ROSCOE) \\ HYDROALCOHOLIC EXTRACT ON THE FUNGUS Lasiodiplodia theobromae
}

\begin{abstract}
The objective is to look for alternative methods to the use of pesticides in large-scale agricultural plantations. Thus, to find an alternative to less aggressive field disease control, alternative methods appear using plant extracts that have antifungal properties. Among these plants is ginger, a species of medicinal, aromatic and spice importance that belongs to the Zingiberaceae family. It is questioned whether the application of hydroalcoholic and aqueous extracts of ginger (Zingiberofficinale [WILLD.] Roscoe) may interfere with the physiological activities of Lasiodiplodia theobromae fungus in vitro. The research methodology was exploratory, descriptive and qualitative / quantitative. The work was carried out at the Multiple Laboratories I and the Biological Sciences Laboratory of the Maria Milza College (FAMAM), on the Governor Mangabeira campus. The fungal isolate used in the research was obtained from characteristic lesions of cassava root rot. The isolate was stored in Petri dishes containing Potato-Dextrose-Agar (BDA) medium in a refrigerator. The analyzes performed in this research showed that the hydroalcoholic and aqueous extract of ginger at concentrations above $15 \%$ showed the greatest interferences in fungal conidia growth, sporulation and germination, preventing its development in vitro. Due to these promising results, it would be interesting to perform other analyzes considering this system factor (ginger extract and the fungus L. theobromae) analyzing other parameters that may serve as a confirmatory basis of the fungitoxic action of ginger extract.

Keywords: Pesticide. Poisoning. Plant extracts. Ginger. Herbal medicine.
\end{abstract}



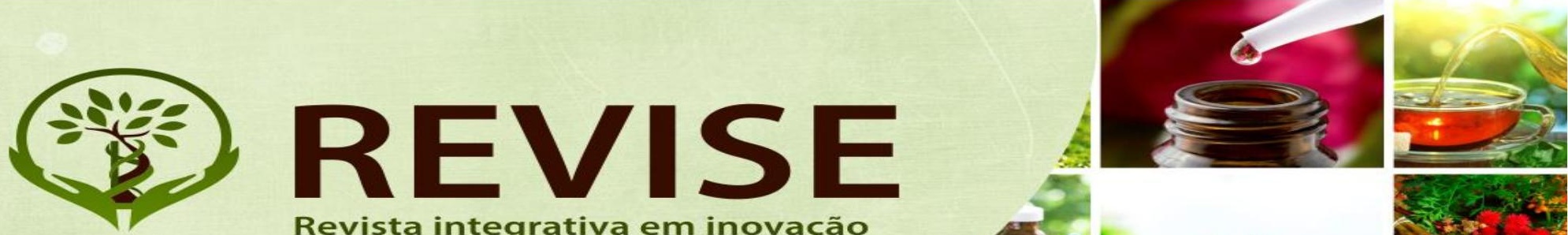

Revista integrativa em inovação tecnológica nas ciências da saúde

Introdução: A sociedade moderna vem questionando o uso de agrotóxico no controle de doenças na agricultura, em virtude dos possíveis efeitos maléficos ao ser humano, além de ter promovido inúmeros problemas ambientais, que podem estar associado à contaminação do solo, água, alimentos, animais e intoxicações dos agricultores. Assim, na tentativa de encontrar uma alternativa ao controle de doenças no campo, menos agressivo, surge métodos alternativos utilizando extratos de plantas que apresentam propriedades antifúngicas. Dentre estas plantas, se encontra o gengibre, uma espécie de importância medicinal, aromática e condimentar que pertencente à família Zingiberaceae.

Problemática geral: A aplicação dos extratos hidroalcoólico e aquoso de gengibre (Zingiberofficinale [WILLD.] Roscoe) podem interferir nas atividades fisiológicas do fungo Lasiodiplodia theobromae in vitro?

Problemática específica: Qual percentagem de inibição do crescimento micelial, da esporulação e germinação de conídios do fungo L. theobromae com a aplicação do extrato hidroalcoólico e aquoso de gengibre nas concentrações de 5, 10, 15 e 25\%? Quais as interferências na fisiologia do fungo L. theobromae com o uso destes extratos? Qual o melhor tipo de extrato? Qual a concentração mais efetiva?

Fundamentação teórica: Em consequência dos prejuízos que os agroquímicos vêm ocasionando a sociedade, prejudicando a saúde humana e causando alterações nos ciclos biológicos do meio ambiente, tem aumentado a busca por produtos alternativos no controle de doenças e pragas na agricultura (MAMPRIM, 2011).

Esta tendência do mundo tem direcionado para a diminuição na agricultura da aplicação dos produtos químicos. Vários métodos alternativos vêm sendo usados especialmente pelos produtos orgânicos e pelos produtores convencionais no combate as pragas, procurando diminuir os custos de produção e especialmente os danos ocasionados ao meio ambiente (LOPES et al., 2004).

Desde a antiguidade o homem mundialmente faz uso de produtos naturais com fins paliativos, profiláticos e curativos no tratamento de doenças. Ainda nas civilizações antigas 

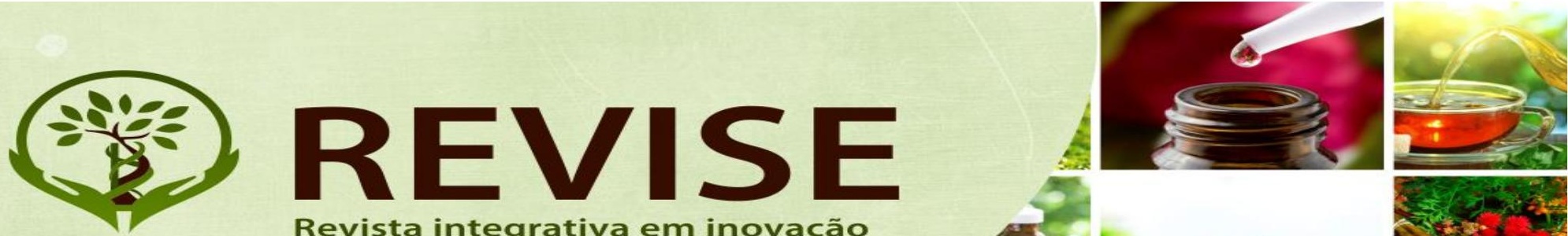

Revista integrativa em inovação tecnológica nas ciências da saúde

ISSN: 2179-6572

houve indícios de plantas medicinais e tóxicas no tratamento de doenças servindo de importante fonte de compostos biologicamente ativos (ANDRADE; CARDOSO; BASTOS, 2007; GOMES et al., 2007).

Vários métodos alternativos, a base de compostos vegetais, vêm sendo usados especialmente pelos produtos orgânicos e pelos produtores convencionais no combate as pragas, procurando diminuir os custos de produção e especialmente os danos ocasionados ao meio ambiente (LOPES et al., 2004). Há milhares de anos utilizam-se os fungicidas obtidos a partir dos princípios ativos das plantas, o que em consequência, devido a sua facilidade de uso, tem se aumentado os estudos a procura de fungicidas originados de plantas. Devido à eficácia, tem sido relatado na literatura, por meio de estudos in vitro, em que vários patógenos são controlados, através de extratos vegetais (SOUZA et al., 2007).

O gengibre é uma espécie que pertence à família Zingiberaceae, esta família possui 47 gêneros e 1400 espécies, adotada no mundo inteiro, cuja designação latina é Zingiber officinalee grega: Zinziberi, o gênero Zingiber possui aproximadamente 150 espécies espalhada especialmente em regiões tropicais e subtropicais da Ásia, essa especiaria foi descrito por William Roscoe no ano de 1807, caracterizado atualmente como Zingiber officinale [Willd.] Roscoe (VASALA, 2004a; PALATTY et al., 2013; RAVINDRAN et al., 2005).

Os rizomas do gengibre trazem grandes benefícios para a saúde, podendo ser usados para tratar várias doenças e perturbações da saúde, tendo suas propriedades farmacológicas destinadas ao tratamento dos distúrbios gastrointestinais como diarreia, vômitos, náuseas, dores de estômagos, flatulência e úlceras gástricas (USHA; KRISHNAPURA, 2009).

Foi através da busca de alternativos que não fosse agressivo ao meio ambiente que os extratos de plantas vêm sendo usado com muito sucesso no controle de fungos fitopatogênicos (SILVA et a., 2006). Neste sentido, estudos tem demonstrado a potencialidade dos extratos de gengibre para o combate de fitopatógenos, principalmente fungos, a exemplo de Rhizoctonia solani, Aspergillus sp., Colletotrchum gloeosporioides, Fusarium sp. e Macrophomina phaseolina (SAJU; VENUGOPAL; MATHEW, 1998; RAJA; KURUCHEVE, 1998; SINGH; RAI, 2000).

Avaliação in vitro. Revista Revise, vol 3, $n^{o}$ fluxo contínuo, p. 80-87. 

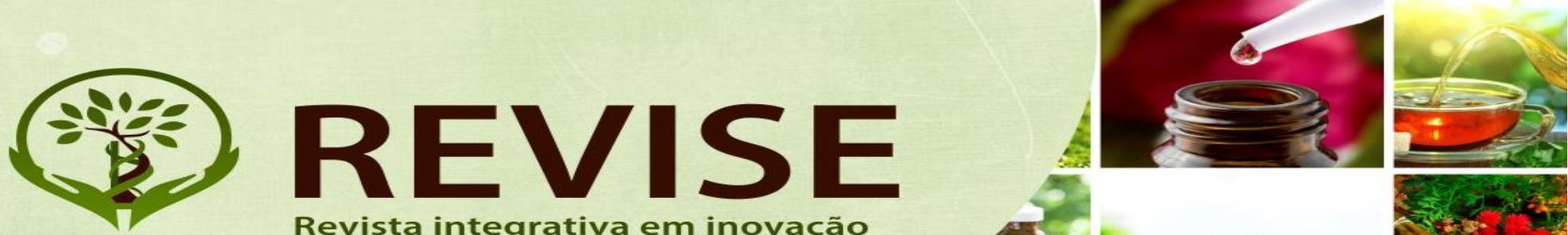

Revista integrativa em inovação tecnológica nas ciências da saúde

ISSN: $2179-6572$
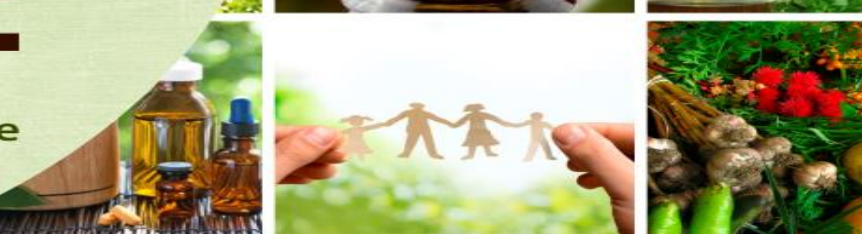

Métodos: A metodologia empregada foi de caráter exploratório descritivo e de natureza qualitativa e quantitativa

Participantes:Participaram da pesquisa três estudantes de graduação (Bacharelado em Biomedicina, Farmácia e Enfermagem) e dois professores doutores em Agronomia e Ciências Agrárias.

Instrumentos: $\mathrm{O}$ crescimento micelial foi medido através do diâmetro de colônias, a contagem de esporulação e conídios germinados foi feita com o auxílio do hemacitrômetro, as análises foram feitas por meio das fórmulas Brito; Nascimento, 2015.

Procedimentos: O trabalho foi realizado nos Laboratórios Múltiplo I e no de Ciências Biológicas da Faculdade Maria Milza (FAMAM), no campus de Governador Mangabeira.

O isolado fúngico utilizado na pesquisa foi obtido a partir de lesões características da podridão da raiz da mandioca. O isolado foi conservado em placas de Petri contendo o meio Batata-Dextrose-Ágar (BDA) em geladeira.

Na preparação dos extratos, foi utilizado $100 \mathrm{~g}$ do material vegetal (rizomas frescos de gengibre - Z. officinale), que foram triturados em liquidificador com álcool etanólico, colocados em um recipiente de vidro e submetidos, por um período de 96 horas, ao processo de extração por infusão. Posteriormente, o extrato ficou em recipiente aberto e em seguida filtrado e submetido á radiação ultravioleta, de acordo com metodologia de Brito; Nascimento (2015). O extrato obtido foi armazenado em refrigerador a $4^{\circ} \mathrm{C}$ para o uso subsequente nos ensaios em laboratório. Para a produção do extrato aquoso foi apenas utilizado água estéril. Após triturados em liquidificador, filtrados e mantidos em UV, foram imediatamente utilizados nas avaliações in vitro.

Os extratos hidroalcoólico e aquoso de gengibre foram avaliados determinando-se a percentagem de inibição e o diâmetro do crescimento micelial em placas de Petri contendo o meio BDA, acrescido das diferentes concentrações do extrato. Ao meio BDA fundente $\left(45^{\circ} \mathrm{C}\right)$ foi adicionado os tratamentos em estudo nas diferentes concentrações (5, 10, 15 e 20\%), e vertidos para placas de Petri, sendo o apenas o meio BDA para a testemunha.

Realizou-se a determinação da produção de conídios após a avaliação do crescimento micelial. Para o preparo da suspensão de conídios, foi adicionado $20 \mathrm{ml}$. de água destilada 

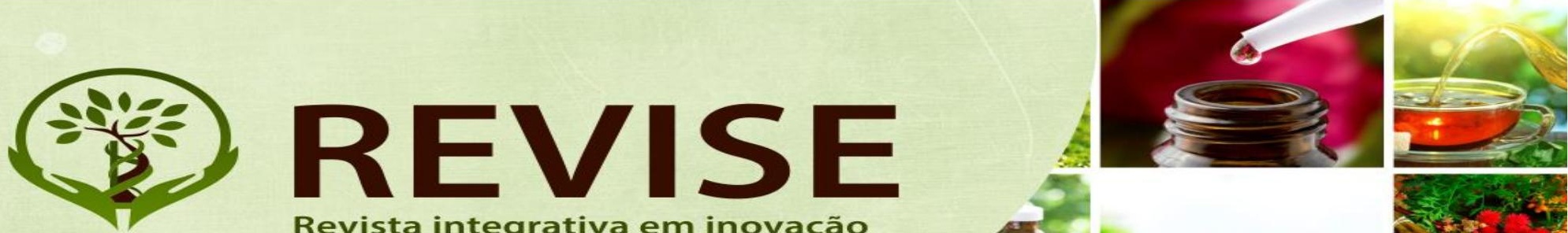

Revista integrativa em inovação tecnológica nas ciências da saúde

ISSN: 2179-6572

esterilizada (ADE) nas placas contendo cada tratamento. Utilizou-se uma escova de cerdas macias para facilitar a remoção do micélio do fungo. O material foi filtrado em duas camadas de gaze esterilizada e a concentração determinada em hemacitômetro, com microscópio óptico, obtendo-se média de cinco leituras para cada um dos tratamentos (BRITO; NASCIMENTO, 2015).

Para a germinação de conídios, $1,0 \mathrm{~mL}$ de uma solução de conídios do fungo $L$. theobromae na concentração de $\left(1,94 \times 10^{6}\right)$ foi adicionada a tubos de ensaio contendo 1,0 $\mathrm{mL}$ do extrato em diferentes concentrações e para a testemunha apenas a solução do fungo com ADE. A avaliação foi realizada com 24 h após a incubação em câmara de crescimento do tipo BOD, a $25 \pm 2^{\circ} \mathrm{C}$.

Análises de dados: Os dados foram analisados por estatística descritiva utilizando-se o delineamento estatístico aleatório em esquema fatorial. Foram analisadas as medidas de tendência central (média) e de variabilidade dos dados (desvio padrão e coeficiente de variação). As análises de variância (teste F) através da análise de regressão, com o auxílio do "software SISVAR" (FERREIRA, 2000).

Resultado: Nesta análise observa-se a interação significativa entre as concentrações do extrato hidroalcoólico de gengibre e os dias de avaliação. Houve uma maior influência das concentrações do extrato hidroalcoólico de gengibre sobre o crescimento micelial do fungo durante as avaliações, tendo percentual de inibição (PIC) de $98 \%$, quanto o extrato aquoso apresentou inibição de 95\%. Diante da esporulação e da germinação os extratos apresentaram percentuais iguais, tendo PIE, 99\% e PIG, 94\%.

Discussão: Os extratos aquosos e hidroalcoólico de gengibre apresentaram ação fungitóxica sobre o desenvolvimento do fungo, interferindo diretamente no crescimento micelial, na esporulação e na germinação de conídios. Resultados semelhantes foram observados por Brito e Nascimento (2015) ao utilizarem o extrato de gengibre no controle de Curvularia eragrostidis (P. Henn.) Meyer, perceberam que este tinha efeito fungitóxico sobre o fungo. Em estudo realizado por Rodrigues et al., (2006) avaliando o extrato de gengibre, observaram que o extrato interferiu na esporulação do fungo Helminthosporum sp. em bananeira. Os extratos de gengibre e alho também se mostraram efetivos sobre $C$.

Avaliação in vitro. Revista Revise, vol 3, $n^{o}$ fluxo contínuo, p. 80-87. 

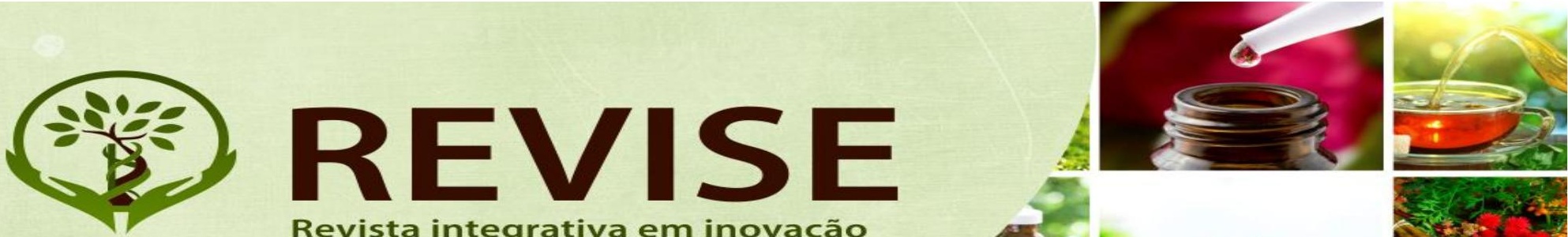

Revista integrativa em inovação tecnológica nas ciências da saúde

ISSN: 2179-6572

eragrostides, pois quando se aumentava a concentração do aumentava progressivamente a percentagem de inibição, mostrando alta eficiência fitotóxica sobre as atividades biológicas do fungo. Araújo et al. (2009), avaliou a ação fungitóxica de extratos de gengibre sobre os fungos Aspergillus ochraceus, Penicillium roqueforti e Rhizopus stolonifer e demonstraram que os extratos alcoólicos de plantas desidratadas a 10, 20 e $25 \%$ controlou $100 \%$ do crescimento micelial dos fungos.

As análises realizadas nesta pesquisa mostraram que o extrato hidroalcoólico e aquoso de gengibre nas concentrações acima de $15 \%$ mostraram as maiores interferências no crescimento, na esporulação e germinação de conídios do fungo, impedindo o seu desenvolvimento in vitro.

Em virtude destes resultados promissores, seria interessante a realização de outras análises levando em consideração este fator sistema (extrato de gengibre e o fungo $L$. theobromae) analisando outros parâmetros que possam servir de base confirmatória da ação fungitóxica do extrato de gengibre.

\section{Referências:}

ANDRADE, S.F.; CARDOSO, L.G.; BASTOS, J.K. Anti-inflammatory and antinociceptive activities of extract, fractions and populnoic acid from bark wood of Austroplenckia populnea. Journal of Ethno Pharmacoly, v.109, n. 3, p. 464-471, 2007.

ARAUJO, R. DE C.Z.; Avaliação in vitro da atividade fungitóxica de extratos de condimentos na inibição de fungos isolados de pães. Ciências e Agrotecnologia. V. 33, n. 2. Lavras, Mar/abr. 2009

BRITO, N. M.; NASCIMENTO, L. C. Potencial fungitóxico de extratos vegetais sobre Curvularia eragrostidis (P. Henn.) Meyer in vitro. Revista Brasileira de Plantas Medicinais, Campinas, v.17, n.2, p.230-238, 2015.

FERREIRA, D. F. Análises estatísticas por meio do SISVAR para Windows versão 4.0. In: Reunião anual da região brasileira da sociedade internacional de biometria, 45. São Carlos, SP, 2000. Programas e Resumos... São Carlos: UFSCar, p.235, 2000.

LOPES, P. S. N. et al. Controle Fitossanitário Alternativo em Comunidades de Pequenos Produtores Rurais no Norte de Minas Gerais. Universidade Federal de Minas 

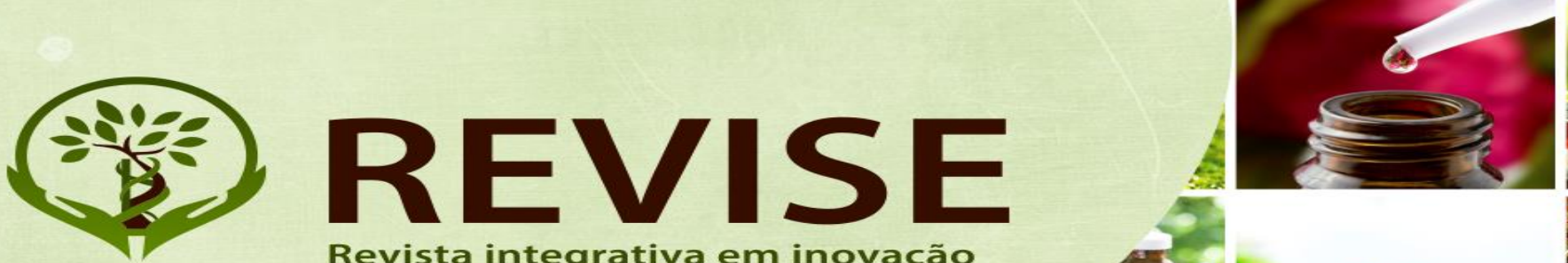

Revista integrativa em inovação tecnológica nas ciências da saúde

ISSN: 2179-6572

Gerais-UFMG. Anais do $2^{\circ}$ Congresso Brasileiro de Extensão Universitária. Belo Horizontesetembro de 2004 .

MAMPRIM, A. P. Efeitos de defensivos agrícolas naturais e extratos vegetais sobre parâmetros biológicos de Metarhiziumanisopliae (Metsch.) Sorok. Marechal Cândido Rondon. 2011.

PALATTY P.L., HANIADKA R., VALDER B., ARORA R. E M.S., B. Ginger in the prevention of nausea and vomiting: a review Crit Rev Food Sci Nutr, v. 7, p. 659-669. 2013.

RAJA, J.; KURUCHEVE, V. Influence of plant extracts and buffalo urine on the growth and sclerotial germination of Macro phomina phaseolina. Indian Phytopathology, New Delhi, v. 51, n. 1, p.102-103, 1998.

RAVINDRAN, P.N.; NIRMAL BABU, K. Ginger: the genus zingiber. Washington: CRC Press, 2005.

RODRIGUES, E. et al. Avaliação da atividade antifúngica de extratos de gengibre e eucalipto in vitro e em fibras de bananeira infectadas com Helminthos porium sp. Acta Scientiarum Agronomy, v.28, n.1, p.123-7, 2006.

SAJU, K A.; VENUGOPAL, M. N.; MATHEW, M. J. Antifungal and insectrepellent activities of essential oil of turmeric (Curcuma longa L.). Current Science, Bangalore, v.75, n.7, p.660-662, 1998.

SINGH, R.; RAI, B. Antifungal potential of some higher plants against Fusarium udum causing wilt disease of Cajanus cajan. Microbios, Cambridge, v.102, n.403, p.165$173,2000$.

SOUZA, A. E. F.; ARAÚJO, E.; NASCIMENTO, L. C. Atividade antifúngica de extratos de alho e capim-santo sobre o desenvolvimento de Fusarium proliferatum isolado de grãos de milho. Fitopatologia Brasileira, Brasília, v.32, n.6, p.465-471, 2007.

USHA, N. S. P. e KRISHNAPURA, S. Gastrointestinal protective effect of dietary spices during ethanol-induced oxidant stress in experimental rats. Physiol. Nutr. Metab. p. 134-141, 2009.

VASALA, P. A. Handbook of herbs and spices Cambridge, UK, Wood head Publishing, 2004a.

Avaliação in vitro. Revista Revise, vol 3, $n^{o}$ fluxo contínuo, p. 80-87. 